\title{
Hydrodynamic Trapping of Swimming Bacteria by Convex Walls
}

\author{
O. Sipos, ${ }^{1}$ K. Nagy, ${ }^{1}$ R. Di Leonardo, ${ }^{2,3}$ and P. Galajda ${ }^{1, *}$ \\ ${ }^{1}$ Institute of Biophysics, Biological Research Centre of the Hungarian Academy of Sciences, H-6726 Szeged, Hungary \\ ${ }^{2}$ Dipartimento di Fisica, Università La Sapienza, P. le A. Moro 2, I-00185 Rome, Italy \\ ${ }^{3}$ NANOTEC-CNR, Institute of Nanotechnology, Soft and Living Matter Laboratory, Piazzale A. Moro 2, I-00185 Rome, Italy
}

(Received 12 February 2015; revised manuscript received 21 May 2015; published 25 June 2015)

\begin{abstract}
Swimming bacteria display a remarkable tendency to move along flat surfaces for prolonged times. This behavior may have a biological importance but can also be exploited by using microfabricated structures to manipulate bacteria. The main physical mechanism behind the surface entrapment of swimming bacteria is, however, still an open question. By studying the swimming motion of Escherichia coli cells near microfabricated pillars of variable size, we show that cell entrapment is also present for convex walls of sufficiently low curvature. Entrapment is, however, markedly reduced below a characteristic radius. Using a simple hydrodynamic model, we predict that trapped cells swim at a finite angle with the wall and a precise relation exists between the swimming angle at a flat wall and the critical radius of curvature for entrapment. Both predictions are quantitatively verified by experimental data. Our results demonstrate that the main mechanism for wall entrapment is hydrodynamic in nature and show the possibility of inhibiting cell adhesion, and thus biofilm formation, using convex features of appropriate curvature.
\end{abstract}

Self-propelled bacteria living in aqueous media have constant, vivid interactions with their local environment, which may dramatically alter their swimming behavior [1-8]. Often, bacterial habitats are physically confined by solid boundaries. Physical interactions with these solid surfaces give rise to a rich variety of dynamical phenomena like steering or rectification of swimming direction [9] and the propulsion of microfabricated structures [10]. Understanding the physical mechanisms behind cell-surface interactions is of crucial importance to design structures that could fully exploit those effects for microfluidic applications. On the other hand, a quantitative understanding of wall entrapment and subsequent adhesion would allow us to design surfaces that hinder unwanted biological processes like biofilm formation. Surface colonization by biofilm-forming bacteria is initiated by cell contact and adhesion to the surface [11-13]. The subsequent biofilm growth can cause highly resistant bacterial infections on medical implants and catheters or impaired industrial equipment [14-19].

Using a tracking microscope, Frymier et al. observed that bacteria display a marked tendency to swim adjacent to wall surfaces [20]. At the beginning, this behavior was attributed to an attractive interaction potential between the cell body and the solid surface. It was later proposed, however, that, while DLVO forces could be responsible for irreversible adhesion, wall entrapment during swimming may have a purely hydrodynamic origin [21,22]. Hydrodynamic effects can indeed give rise to wall entrapment via two distinct mechanisms. The first one is via farfield, dipolar flows that, once reflected by a flat wall, give rise to reorientation parallel to the wall surface as well as an attraction by the wall [23]. The second mechanism involves hydrodynamic torques that arise in anisotropic bodies swimming in close contact with the wall and leading to swimming at a finite angle with the wall surface [22]. More recently, the role of hydrodynamic interactions has been questioned [24,25], suggesting that steric repulsion and rotational Brownian motion are enough to reproduce the observed accumulation of bacteria in the proximity of solid walls. Despite the extensive theoretical and numerous experimental works on the hydrodynamics of bacterial swimming, a straightforward, unambiguous, and direct identification of a main mechanism responsible for wall entrapment is still lacking. In a way, all these models capture different aspects of the problem and are all capable of justifying the accumulation effects observed over flat walls. While concave confining walls will tend to stabilize trapping [26], what happens if the wall is convex? Will all proposed mechanisms for wall entrapment still give the correct answer when the walls are curved? Can we break entrapment at some critical curvature radius and inhibit cell adhesion by using only geometry?

In this Letter, we study bacterial entrapment by microfabricated convex surfaces. The trajectories of swimming bacteria display a marked tendency to follow the wall curvature, although entrapment by convex walls is progressively reduced below a characteristic radius. Our results demonstrate that the main mechanism for wall entrapment is hydrodynamic in nature and involves a finite swimming angle that keeps the cell in close contact with bounding surfaces. If the surface is convex, the swimming angle is progressively reduced for increasing surface curvature up to a critical curvature radius where entrapment becomes 
unstable. As a direct consequence of that, we show that cell colonization of solid walls can be strongly inhibited by choosing convex surfaces with a curvature radius below a characteristic size.

The polydimethylsiloxane (PDMS) microfluidic devices were fabricated by using soft lithography techniques [27,28]. (See Supplemental Material [29] for a more detailed materials and methods.) Each device was attached to a glass coverslip by oxygen plasma treatment. The microfluidic chips contained $75 \mu \mathrm{m}$ high straight walls and pillars with circular bases of different radii $(20-350 \mu \mathrm{m})$. The devices were filled with a suspension of smooth swimming E. coli cells of the HCB437 strain [30] carrying the pMPMA2-GFPmut2 plasmid [31]. Bacteria were imaged at 10 frames per second by an epifluorescence microscope equipped with $10 \times$ and $40 \times$ objectives and an EM-CCD camera. Bacterial trajectories were considered to be tracing the solid surface of the walls and pillars if the tracing time was longer than 30 frames (3 s) [32], and the cells remained in the $3.5 \mu \mathrm{m}$ vicinity of the surface.

We tracked and analyzed 1124 bacterial trajectories along the walls and the perimeter of the pillars in microchambers (Fig. 1; see Supplemental Material [29] for an additional image of the microfluidic chip). Most of these cells indeed followed the curvature of the pillars, indicating the presence of a hydrodynamic trapping mechanism acting on the swimming cells [Fig. 2(a)]. It is known that bacteria tend to swim in clockwise circles over a solid flat wall [33-37]. In order to exclude interaction with the bottom surface as the source of the observed curved trajectories, we included in the statistical analysis only those bacteria that circulate around the pillars in an anticlockwise direction. We found that more than $90 \%$ of the cells colliding to a planar wall swam along its surface. At pillars with radii larger than $50 \mu \mathrm{m}, 60 \%-90 \%$ of the cells swam around. Below $50 \mu \mathrm{m}$ radius, the tracing cell fraction seems to decrease with the decreasing pillar radius. However, the trapping effect was able to keep some bacteria near the wall
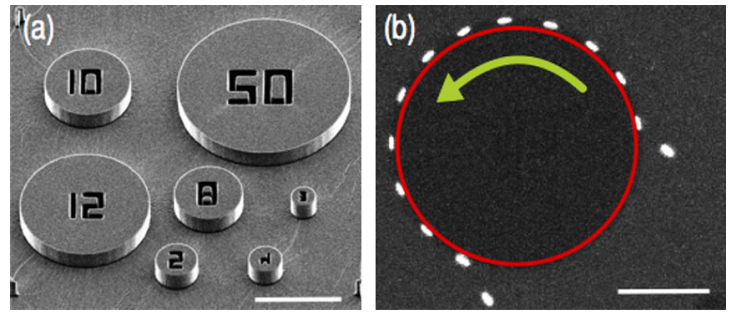

FIG. 1 (color online). (a) A scanning electron micrograph of the PDMS-based microdevice containing several micropillars. The height of the posts is $75 \mu \mathrm{m}$. The scale bar is $250 \mu \mathrm{m}$. (b) A montage of fluorescence microscopy images of a bacterial cell following the curved surface of a micropillar. The time between each position of the cell shown is $2.5 \mathrm{~s}$. The arrow indicates the swimming direction of the cell. The perimeter of the pillar is indicated by a solid red line. The scale bar is $100 \mu \mathrm{m}$. even in the case of the smallest radius of curvature $(20 \mu \mathrm{m})$. The existence of a characteristic pillar radius below which the trapping probability decreases can be explained by using a simple hydrodynamic model. We model a swimmer as the combination of a spherical cell body and a helical flagellar bundle that are rigidly connected as shown in Fig. 3. We neglect hydrodynamic interactions between the cell body and flagellar bundle. Since our main focus here is on hydrodynamic entrapment to the wall, we constrain cell motions on the $x, y$ plane and neglect $z$ motions that arise from axial rotations of both the cell body and flagellar bundle. When the cell body "hits" a wall, a normal reaction force develops that constrains body motions to the $x$ direction. From the linearity of Stokes equation, the viscous torque acting on the cell body will be linearly connected to the instantaneous values of rotational and translational velocities. Having restricted the motion of the cell on the $x, y$ plane and as long as the cell moves in contact with the wall, we are left with only two velocity components: the $x$ component of linear speed $U$ and the $z$ component of angular speed $\Omega$. Calling $T^{b}$ the $z$ component of the viscous torque acting on the cell body, we can write

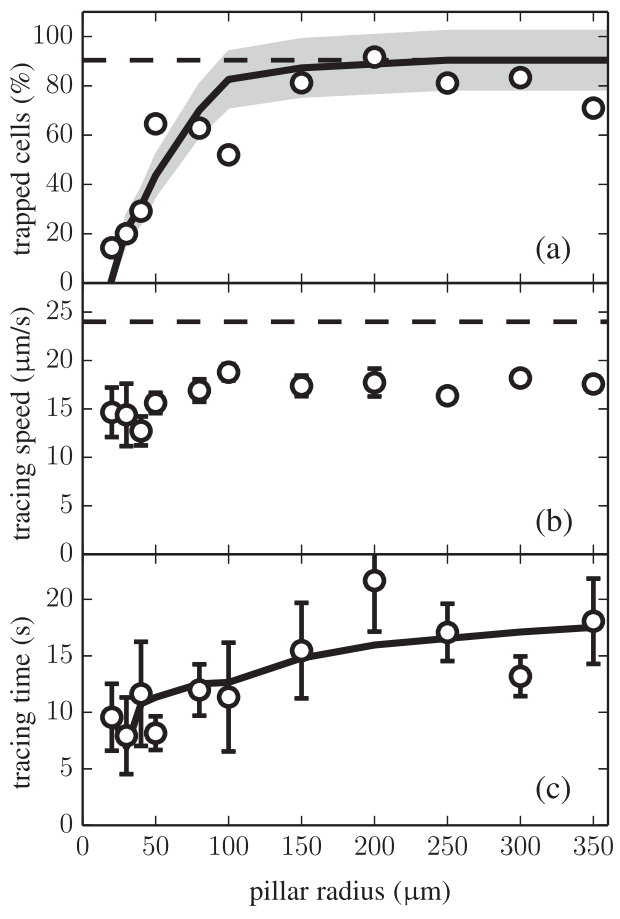

FIG. 2. (a) The measured ratio of the cells that followed the wall of a pillar after collision (open circles) and the model prediction for the fraction of trapped cells (solid line). The dashed line represents the value for flat walls. The gray shaded area extends for one standard deviation above and below the predicted result. (b) The average tracing speed of bacteria near pillars. The dashed line represents the average speed in bulk. (c) The measured average residence time of bacteria for each pillar (open circles) and the model prediction (solid line). The error bars indicate standard errors. 


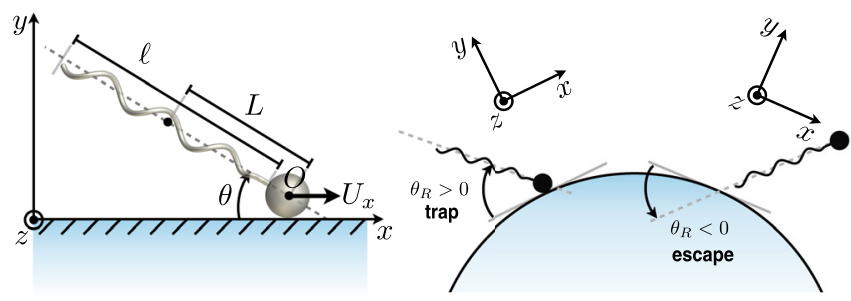

FIG. 3 (color online). A schematic illustration and notation for the hydrodynamic model proposed in the text.

$$
T^{b}=-C^{b} \Omega-B^{b} U
$$

The first term $-C^{b} \Omega$ represents the viscous drag that would act on the cell body if purely rotating. The value of $C^{b}$ is affected by the presence of the wall, and it would be nonzero even in the bulk. The coupling term $-B^{b} U$ represents the viscous torque acting on the cell body when purely translating. The value of $B^{b}$ is zero in the bulk and becomes positive nearby a wall due to the higher shear rates in the gap region. The resistance coefficients $C^{b}$ and $B^{b}$ depend on the origin we choose for torque evaluation. Here and in the following, we set the origin in the cell body center $O$ and choose the signs so that all resistance coefficients are positive. Similarly, the torque on the flagellar bundle will display, in addition to a rotational drag proportional to $\Omega$, a coupling term connected to the linear speed $U$ :

$$
T^{f}=-C^{f} \Omega+B^{f} U \text {. }
$$

The coupling term is now positive and arises from the displacement of the bundle center of resistance from the origin $O$ :

$$
B^{f}=L \gamma_{\perp} \sin \theta
$$

where $\gamma_{\perp}$ is the drag coefficient determining the viscous resistance encountered by the flagellar bundle when translating in a direction perpendicular to its axis. The only external force is represented by the reaction force from the wall. The normal component of that force will not produce any torque about our origin $O$. We cannot exclude, in principle, the presence of a tangential friction component, but it will just add up to the hydrodynamic component $B^{b}$. The overall system is therefore torque-free so that we can add Eqs. (1) and (2) and solve for $\Omega$ :

$$
\Omega=\frac{B^{f}-B^{b}}{C^{b}+C^{f}} U=\frac{L \gamma_{\perp} \sin \theta-B^{b}}{C^{b}+C^{f}} U .
$$

The swimming angle $\theta$ has a stable equilibrium value $\theta_{\infty}$ for which $\dot{\theta}=-\Omega=0$ :

$$
\sin \theta_{\infty}=\frac{B^{b}}{L \gamma_{\perp}}
$$

where the subscript $\infty$ indicates that we are here considering the case of flat wall or, in other words, an infinite pillar radius $R$. Pillar radii are always enough larger than the cell size so that we can assume that all hydrodynamic resistance matrices are negligibly affected by the small wall curvature. In that situation, we can take into account the finite curvature of the pillar by moving to a reference frame that rotates around the pillar axis with an angular speed given by $-U / R$. In this new reference frame, the time derivative of $\theta$ will be given by

$$
\dot{\theta}=-\Omega-\frac{U}{R}=\left(\frac{B^{b}-L \gamma_{\perp} \sin \theta}{C^{b}+C^{f}}-\frac{1}{R}\right) U .
$$

The stable value for $\theta$ now decreases as the pillar radius becomes smaller:

$$
\sin \theta_{R}=\sin \theta_{\infty}-\frac{C^{b}+C^{f}}{L R \gamma_{\perp}}
$$

There exists a critical value $R^{*}$ for the pillar radius below which there is no positive stable value for $\theta ; \dot{\theta}$ will still be negative even when $\theta=0$, so that the cell will eventually swim away from the surface (Fig. 3):

$$
R^{*}=\frac{C^{b}+C^{f}}{L \gamma_{\perp} \sin \theta_{\infty}} .
$$

A first estimate of the critical radius can be obtained by noting that $C^{f} \gg C^{b}$ and that $C^{f}=\ell^{2} \gamma_{\perp} / 3$ [35]. Substituting into (8), we obtain

$$
R^{*} \approx \frac{\ell^{2}}{3 L \sin \theta_{\infty}} \approx \frac{2}{3} \frac{\ell}{\sin \theta_{\infty}},
$$

where, in the last passage, we used the fact that the cell length $2 L$ is mostly given by the length of the flagellar bundle $\ell$. As shown in (5), the actual value of $\theta_{\infty}$ depends on $B^{b}$, a quantity that is expected to be very sensitive on the actual value of the gap between the cell and the wall.

If we neglect wall effects on the cell body and set $B^{b}$ to zero as in Ref. [24], we would predict a vanishing swimming angle $\theta_{\infty}$ with cells swimming parallel to flat walls. A vanishing $\theta_{\infty}$ would also lead to a diverging critical radius for trapping and fail to predict stable trapping around curved surfaces. Rather than entering into the difficulties of theoretically predicting $B^{b}$, we prefer to obtain $\theta_{\infty}$ from experimental observations and check the theory by directly verifying relation (9). To this aim, we used video microscopy with higher magnification to determine the cell orientation during swimming near flat obstacles. A total of 58 individual cells were tracked. As 
expected, we found that the cells swim with a finite average swimming angle to the surface plane (Fig. 4; see Supplemental Material [29] for images of a bacterium near a convex surface). The observed average value for $\theta_{\infty}$ is $5^{\circ}$, which for a typical bundle length of $7.5 \mu \mathrm{m}$ gives $R^{*}=57 \mu \mathrm{m}$. The distribution of average $\theta_{\infty}$ is actually very broad and is reported in Fig. 4(b). The observation that most of the cells are trapped by pillars of radii larger than $150 \mu \mathrm{m}$ [Fig. 2(a)] is consistent with a $\theta_{\infty}$ distribution dropping quickly below $2^{\circ}$ (corresponding $R^{*} \sim 140 \mu \mathrm{m}$ ). Using the distribution in Fig. 4(b), we can predict the fraction of trapped cells for pillars of radius $R$ as the fraction of $\theta_{\infty}$ angles whose corresponding values of $R^{*}$ is lower than $R$. The results are shown as a solid line in Fig. 2(a), where we have just rescaled predicted fractions to give the correct limit for infinite radius of curvature. Equations (9) and (5) also show that the critical radius for trapping does not depend on the swimming speed as expected from a purely hydrodynamic treatment. Therefore, pillars do not preferentially trap bacteria based on their speed. However, even in a purely hydrodynamic framework, the swimming speed can be affected by a nearby wall [38], and a weak dependence of speed on wall curvature cannot be excluded. This picture is consistent with our data in Fig. 2(b), where the average swimming speed for trapped cells is plotted as a function of the pillar radius. The measured speeds seem to be quite constantly lower than the average bulk speed $(24 \pm 4 \mu \mathrm{m} / \mathrm{s})$ with possible deviations for radii smaller than $100 \mu \mathrm{m}$. So far, we have considered a very idealized situation where each cell is characterized by a well-defined $\theta_{\infty}$ whose value decides whether a cell escapes from a pillar or is trapped around it forever. However, the actual value of the swimming angle at a confining wall displays large fluctuations with time due to Brownian motion and mostly to cell wobbling. Therefore, even when the average value of $\theta_{R}$ is positive, fluctuations can occur, leading to a negative swimming angle and the consequent escape from the pillar. In this situation, trapped states are expected to show a finite lifetime. The measured average lifetimes of trapped states

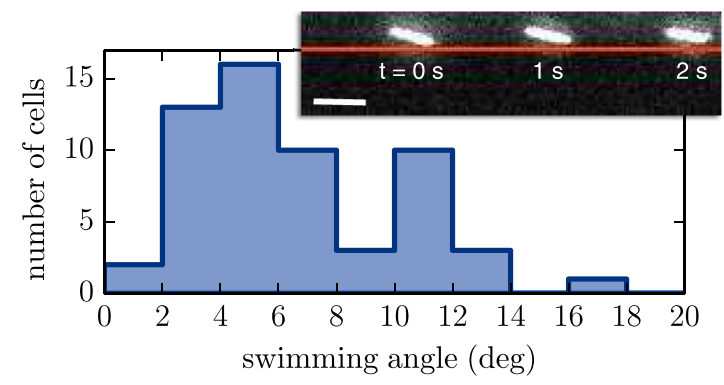

FIG. 4 (color online). A histogram of the average angles of orientation of individual cells with respect to the solid wall $(n=58)$. The inset shows a montage of frames with a swimming bacterium near a planar solid wall. The red line shows the surface of the wall. The scale bar is $5 \mu \mathrm{m}$. are reported in Fig. 2(c) as a function of the pillar radius. We found that bacteria tend to spend more time swimming around pillars with larger radii. This observation can be accounted for by noting that the larger $\theta_{R}$, the larger and more unlikely the fluctuation that is needed to escape from the pillar. Assuming the simple linear relation $\tau=\alpha \theta_{R}$, we can predict the average residence time $(\tau)$ around each pillar from (7) based on the values of $\theta_{\infty}$ in Fig. 4(b). The solid line in Fig. 2(c) shows the predicted average lifetimes corresponding to the best fit value for the phenomenological parameter $\alpha$. It has been shown recently that artificial microswimmers can be captured into sphere-bound orbits by colloidal particles of fixed size [39]. The existence of a critical size for trapping of dipolar swimmers around spherical colloids was recently theoretically predicted by using far-field hydrodynamics [38]. In contrast to what we find here, however, trapped swimmers are found to swim with their axis pointing away from the surface (negative $\theta$ with our sign convention) by an angle that goes to zero in the limit of a flat wall. This discrepancy is probably due to the neglected near-field contributions that, in the case of bacterialike swimmers, tend to reorient the cell in a direction that points into the surface [40].

The observed wall entrapment may have biological importance. The first step of biofilm formation is the sticking of planktonic (swimming or floating) cells to surfaces [11]. This initial process may be greatly affected by hydrodynamic effects arising when a cell moves near a surface. In order to investigate the effect of curvature on cell adhesion, we incubated our device with a bacterial culture inside for 18-24 h. During this, the cells reproduced within the microfluidic channel. We flushed the planktonic cells out of the device and imaged the pillars with the adhered cells [Fig. 5(a)]. In order to quantify the level of cell adhesion, we calculated the average fluorescence intensity per pixel around the perimeter of each pillar [Fig. 5(b)]. A larger bacterial concentration was found around pillars with
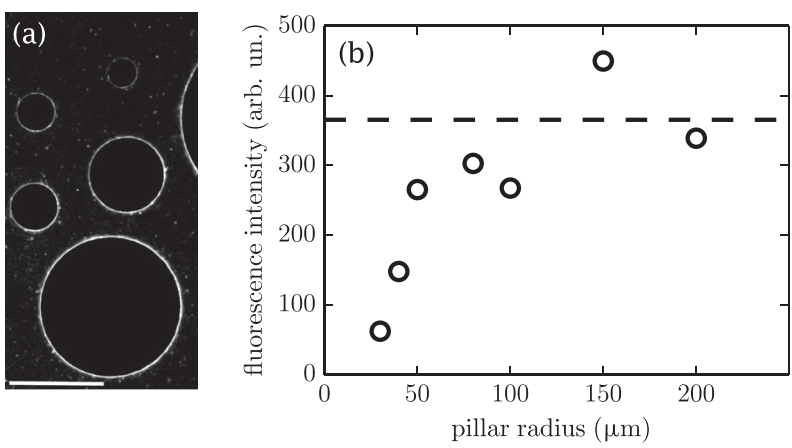

FIG. 5. (a) A representative fluorescence microscopy image showing the bacterial cells adhered to the surface of the micropillars. The scale bar is $200 \mu \mathrm{m}$. (b) The fluorescence pixel intensity over a unit area in the vicinity $(3 \mu \mathrm{m})$ of pillars and next to a planar wall. The dashed line represents the value for flat walls. 
larger radii. This suggests that cells are more likely to attach to more flat surfaces as they spend a longer time around their walls. Bacterial concentration around a pillar shows a dependence on the radius that is very similar to what was previously described for the fraction of trapped cells (Fig. 2).

We studied the swimming motion of $E$. coli cells near convex surfaces to elucidate the main mechanisms behind wall entrapment. We showed that cells orient at an angle so that they swim against the surface while moving along it. As a result, swimming cells can also be trapped by round pillars and follow the convex curvature of the pillar surface. However, we found that this tendency is markedly reduced below a critical radius of curvature of about $50 \mu \mathrm{m}$. These observations are quantitatively reproduced by a simple hydrodynamic model that connects the critical radius for entrapment to the swimming angle over flat walls. Finally, we showed that surface curvatures can strongly affect bacterial colonization in growing cultures, suggesting that a suitable surface geometry may inhibit biofilm formation.

This work has been supported by the "Momentum" program of the Hungarian Academy of Sciences. R. D. L. acknowledges funding from the European Research Council under the European Union's Seventh Framework Programme (FP7/2007-2013)/ERC Grant Agreement No. 307940. We are grateful to K. Fahrner and H. C. Berg for providing the $E$. coli strains.

*galajda.peter@brc.mta.hu

[1] K. V. Thimann and G. M. Curry, Comp. Biochem. 1, 243 (1960).

[2] D-P. Häder, J. Photochem. Photobiol., B 1, 385 (1988).

[3] R. Blakemore, Science 190, 377 (1975).

[4] H. Salman, A. Zilman, C. Loverdo, M. Jeffroy, and A. Libchaber, Phys. Rev. Lett. 97, 118101 (2006).

[5] B. L. Taylor, I. B. Zhulin, and M. S. Johnson, Annu. Rev. Microbiol. 53, 103 (1999).

[6] J. Adler, Science 153, 708 (1966).

[7] H. C. Berg, E. coli in Motion (Springer-Verlag, New York, 2003).

[8] J. P. Armitage, Adv. Microb. Physiol. 41, 229 (1999).

[9] P. Galajda, J. Keymer, P. Chaikin, and R. Austin, J. Bacteriol. 189, 8704 (2007).

[10] R. Di Leonardo, L. Angelani, D. Dell'Arciprete, G. Ruocco, V. Iebba, S. Schippa, M. P. Conte, F. Mecarini, F. De Angelis, and E. Di Fabrizio, Proc. Natl. Acad. Sci. U.S.A. 107, 9541 (2010).

[11] M. C. van Loosdrecht, J. Lyklema, W. Norde, and A. J. Zehnder, Microbiol. Rev. 54, 75 (1990).

[12] C. Beloin, A. Roux, and J.-M. Ghigo, Curr. Top. Microbiol. Immunol. 322, 249 (2008).

[13] J. C. Conrad, Res. Microbiol. 163, 619 (2012).
[14] J. W. Costerton, P. S. Stewart, and E. P. Greenberg, Science 284, 1318 (1999).

[15] T-F. C. Mah and G. A. O’Toole, Trends Microbiol. 9, 34 (2001).

[16] B. W. Trautner and R. O. Darouiche, Am. J. Infect. Control 32, 177 (2004)

[17] C. A. Fux, J. W. Costerton, P. S. Stewart, and P. Stoodley, Trends Microbiol. 13, 34 (2005).

[18] T. Mattila-Sandholma and G. Wirtanena, Food reviews international 8, 573 (1992).

[19] S. W. Borenshtein, Microbiologically Influenced Corrosion Handbook (Woodhead, Cambridge, England, 1994).

[20] P. D. Frymier, R. M. Ford, H. C. Berg, and P. T. Cummings, Proc. Natl. Acad. Sci. U.S.A. 92, 6195 (1995).

[21] M. A. Vigeant and R. M. Ford, Appl. Environ. Microbiol. 63, 3474 (1997).

[22] M. A. S. Vigeant, R. M. Ford, M. Wagner, and L. K. Tamm, Appl. Environ. Microbiol. 68, 2794 (2002).

[23] A. P. Berke, L. Turner, H. C. Berg, and E. Lauga, Phys. Rev. Lett. 101, 038102 (2008).

[24] G. Li and J. X. Tang, Phys. Rev. Lett. 103, 078101 (2009).

[25] G. Li, J. Bensson, L. Nisimova, D. Munger, P. Mahautmr, J. X. Tang, M. R. Maxey, and Y. V. Brun, Phys. Rev. E 84, 041932 (2011).

[26] I. D. Vladescu, E. J. Marsden, J. Schwarz-Linek, V. A. Martinez, J. Arlt, A. N. Morozov, D. Marenduzzo, M. E. Cates, and W. C. K. Poon, Phys. Rev. Lett. 113, 268101 (2014).

[27] G. M. Whitesides, E. Ostuni, S. Takayama, X. Jiang, and D. E. Ingeber, Annu. Rev. Biomed. Eng. 3, 335 (2001).

[28] O. Sipos, K. Nagy, and P. Galajda, Chem. Biochem. Eng. Q. 28, 233 (2014).

[29] See Supplemental Material at http://link.aps.org/ supplemental/10.1103/PhysRevLett.114.258104 for a detailed description of Materials and Methods and additional experimental data.

[30] A. J. Wolfe, M. P. Conley, T. J. Kramer, and H. C. Berg, J. Bacteriol. 169, 1878 (1987).

[31] M. P. Mayer, Gene 163, 41 (1995).

[32] S. P. Strong, B. Freedman, William Bialek, and R. Koberle, Phys. Rev. E 57, 4604 (1998).

[33] H. C. Berg and L. Turner, Biophys. J. 58, 919 (1990).

[34] W. R. DiLuzio, L. Turner, M. Mayer, P. Garstecki, D. B. Weibel, H. C. Berg, and G. M. Whitesides, Nature (London) 435, 1271 (2005).

[35] E. Lauga, W. R. DiLuzio, G. M. Whitesides, and H. A. Stone, Biophys. J. 90, 400 (2006).

[36] D. Giacché, T. Ishikawa, and T. Yamaguchi, Phys. Rev. E 82, 056309 (2010).

[37] R. Di Leonardo, D. Dell'Arciprete, L. Angelani, and V. Iebba, Phys. Rev. Lett. 106, 038101 (2011).

[38] S. E. Spagnolie, G. R. Moreno-Flores, D. Bartolo, and E. Lauga, Soft Matter 11, 3396 (2015).

[39] D. Takagi, J. Palacci, A. B. Braunschweig, M. J. Shelley, and Jun Zhang, Soft Matter 10, 1784 (2014).

[40] S. E. Spagnolie and E. Lauga, J. Fluid Mech. 700, 105 (2012). 